\title{
Central vaspin administration acutely reduces food intake and has sustained blood glucose-lowering effects
}

\author{
N. Klöting • P. Kovacs $•$ M. Kern • J. T. Heiker • \\ M. Fasshauer • M. R. Schön • M. Stumvoll • \\ A. G. Beck-Sickinger • M. Blüher
}

Received: 16 October 2010 / Accepted: 8 March 2011 /Published online: 5 April 2011

(C) Springer-Verlag 2011

\begin{abstract}
Aims/hypothesis Vaspin (visceral adipose tissue-derived serpin) was first identified as an adipokine in a rat model of type 2 diabetes, in which it is predominantly secreted from visceral adipose tissue. Serum concentrations of vaspin show a food intake-related diurnal variation. We therefore tested the hypothesis that vaspin plays a role in the regulation of food intake.

Methods Vaspin levels in the hypothalamus and human stomach were determined by western blotting. The cere-
\end{abstract}

N. Klöting and P. Kovacs contributed equally to this study.

N. Klöting $\cdot$ M. Kern · J. T. Heiker $\cdot$ M. Fasshauer $\cdot$ M. Stumvoll •

M. Blüher $(\square)$

Department of Medicine, University of Leipzig,

Liebigstr. 20,

04103 Leipzig, Germany

e-mail: bluma@medizin.uni-leipzig.de

N. Klöting $\cdot$ M. Stumvoll

IFB Adiposity Diseases,

Junior Research Group 'Animal models of obesity',

Leipzig, Germany

P. Kovacs

Junior Research Group N06, Interdisciplinary Centre for Clinical

Research (IZKF), University of Leipzig,

Leipzig, Germany

M. R. Schön

Städtisches Klinikum Karlsruhe, Clinic of Visceral Surgery,

Karlsruhe, Germany

A. G. Beck-Sickinger

Institute of Biochemistry, Faculty of Life Sciences,

Pharmacy and Psychology, University of Leipzig,

Leipzig, Germany brospinal fluid concentration of vaspin was measured in five healthy volunteers using an ELISA. Fed 11-week-old female $d b / d b$ mice were given intraperitoneal injections of $1 \mathrm{mg} / \mathrm{kg}$ body weight of vaspin $(n=6)$ or saline $(n=6)$ on experimental days 1, 3 and 4. Changes in food intake and fed plasma glucose concentrations were determined after one intracerebroventricular administration of either $1 \mu \mathrm{g}$ vaspin or artificial cerebrospinal fluid into 11-week-old female $d b / d b(n=8)$ and C57BL/6 mice $(n=8)$ up to 6 days after injection.

Results We detected vaspin in the hypothalamus of $d b / d b$ and C57BL/6 mice and in the cerebrospinal fluid of healthy individuals. Both peripheral and central vaspin administration decrease food intake in obese $d b / d b$ and lean C57BL/6 mice. In $d b / d b$ mice, vaspin treatment is associated with sustained glucose-lowering effects for at least 6 days after injection. In addition, we demonstrated expression of the gene encoding vaspin in the gastric mucosa in humans, and found that this was subject to regional variations.

Conclusions/interpretation Our data suggest a previously unrecognised role of vaspin in the regulation of food intake. We postulate that vaspin inhibits a protease that degrades an anti-orexigenic factor.

Keywords Adipokine $\cdot$ Food intake $\cdot$ Obesity $\cdot$ Stomach expression · Vaspin

$\begin{array}{ll}\text { Abbreviations } & \\ \text { ACSF } & \text { Artificial cerebrospinal fluid } \\ \text { i.c.v. } & \text { Intracerebroventricular } \\ \text { KORA study } & \begin{array}{l}\text { Kooperative Gesundheitsforschung in der } \\ \text { Region Augsburg study }\end{array} \\ \text { Vaspin } & \text { Visceral adipose tissue-derived serpin }\end{array}$




\section{Introduction}

Visceral adipose tissue-derived serpin (vaspin) is a member of the serine protease inhibitor family and is produced in the visceral adipose tissue of Otsuka Long-Evans Tokushima Fatty rats at the age when obesity and insulin plasma concentrations reach a peak [1]. In these rats, serum vaspin levels decreased with worsening of diabetes but could be normalised by insulin or pioglitazone treatment. Consistent with these observations, we found that higher serum vaspin concentrations and increased SERPINA12 mRNA expression in human adipose tissue was associated with obesity, insulin resistance and type 2 diabetes [2, 3]. In the Kooperative Gesundheitsforschung in der Region Augsburg [Cooperative Health Research in the Augsburg Region] (KORA) study, vaspin genotype was found to be significantly associated with type 2 diabetes independently of obesity [4].

Administration of vaspin to obese mice improves glucose tolerance, insulin sensitivity and altered gene expression of candidate genes for insulin resistance [1]. Interestingly, circulating vaspin levels follow a meal-related diurnal variation in humans, similar to that seen for ghrelin [5], suggesting a previously unrecognised role of vaspin in the regulation of food intake. Serum vaspin concentrations show a preprandial rise followed by a significant decline in response to meals, and unscheduled food ingestion after a prolonged fast significantly reduces circulating vaspin levels [5]. Based on these findings we sought to determine the effects of peripheral and central vaspin administration on food intake and blood glucose in $d b / d b$ and C57BL/6 mice.

\section{Methods}

Animal studies All experiments were performed in accordance with the Guide for the Care and Use of Laboratory Animals published by the US National Institutes of Health (NIH Publication No. 85-23, revised 1996) and the rules for animal care of the local government authorities (Bezirksregierung Leipzig, Germany) and were approved by the institution's animal care and use committee. Hypothalamus tissue from $d b / d b(n=5)$ and C57BL/6 $(n=$ 5) mice (Taconic Europe, Bomholt, Denmark) was dissected and homogenised in homogenisation buffer with a Polytron homogeniser (IKA, Staufen, Germany), and western blot analyses were performed by standard methods with an anti-vaspin monoclonal antibody (AdipoGen, Seoul, South Korea) [3].

Human studies Human SERPINA12 mRNA expression was measured as described previously [2] in the stomach explants of five morbidly obese, but otherwise healthy, insulin-sensitive individuals who underwent gastric sleeve resection [6]. Immunostaining of paraffin sections from these stomach samples was performed using an anti-vaspin monoclonal antibody (AdipoGen) as described previously [3]. Vaspin concentration was measured in the cerebrospinal fluid of five healthy individuals who underwent diagnostic lumbar puncture using an ELISA [3]. Both studies were approved by the Ethics Committee of the University of Leipzig (031-2006 and 197-2009). All individuals gave written informed consent before taking part in the study.

Repeated i.p. vaspin treatment Experiments were conducted on pathogen-free 11-week-old female $d b / d b$ mice $(n=12)$ at the animal research facility of the University of Leipzig. Mice had free access to standard chow and water. Mice were randomised, based on both body weight and fed plasma glucose concentration, to receive either vaspin $(n=$ $6)$ or saline $(n=6)$. Fed mice were given i.p. injections of pyrogen-free saline or $1 \mathrm{mg} / \mathrm{kg}$ body weight of vaspin $(08: 00 \mathrm{~h})$ on experimental days 1, 3 and 4 (Fig. 1). Food intake and fed plasma glucose were measured at 08:00 h, immediately before i.p. injections.

Intracerebroventricular vaspin administration Experiments were conducted on eight female $d b / d b$ and eight female C57BL/6 mice at our Animal Research Facility at the University of Leipzig. Mice were kept acclimatised at $22 \pm$ $2{ }^{\circ} \mathrm{C}$, with a $12 \mathrm{~h}$ light-dark cycle, and had free access to food (standard chow) and water. When the mice were 11 weeks old, cannulas were implanted for intracerebroventricular (i.c.v.) stimulation. Mice were randomly divided into two groups: one served as a control $(n=4)$ and the other received i.c.v. vaspin $(n=4)$. After i.c.v. injection, food intake, fed plasma glucose and body weight were monitored daily in both groups of animals over a 7-day period. For i.c.v. vaspin stimulation, cannulas were implanted as described previously [7]. Mice were allowed to recover for 1 week after surgery. For baseline measurements, mice were injected with $2 \mu \mathrm{l}$ artificial cerebrospinal fluid (ACSF) immediately before onset of a dark phase and food intake was measured $24 \mathrm{~h}$ later. After a 1 day break, ACSF injection was repeated. After an additional 1 day break, $1 \mu \mathrm{g}$ vaspin or ACSF (both in a total volume of $2 \mu \mathrm{l}$ ) was injected per mouse and food intake was measured $24 \mathrm{~h}$ later.

\section{Statistical analyses}

Data are shown as mean \pm SD. Expression and phenotype differences were assessed using the Student's $t$ test. Linear 

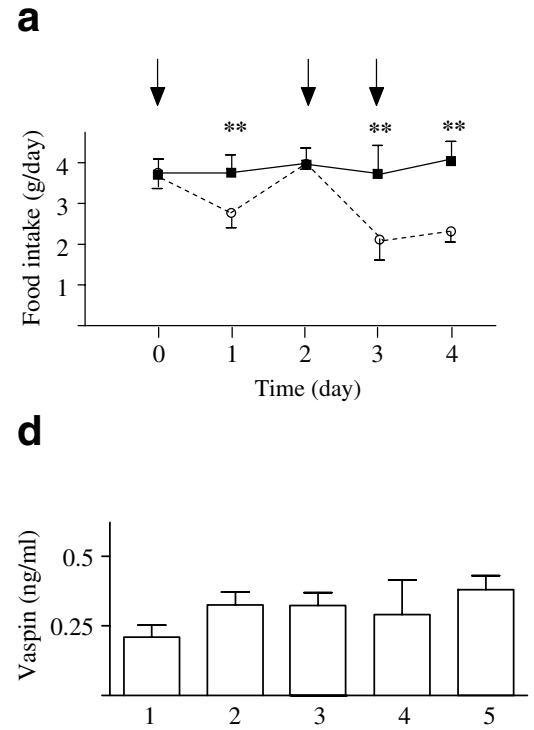
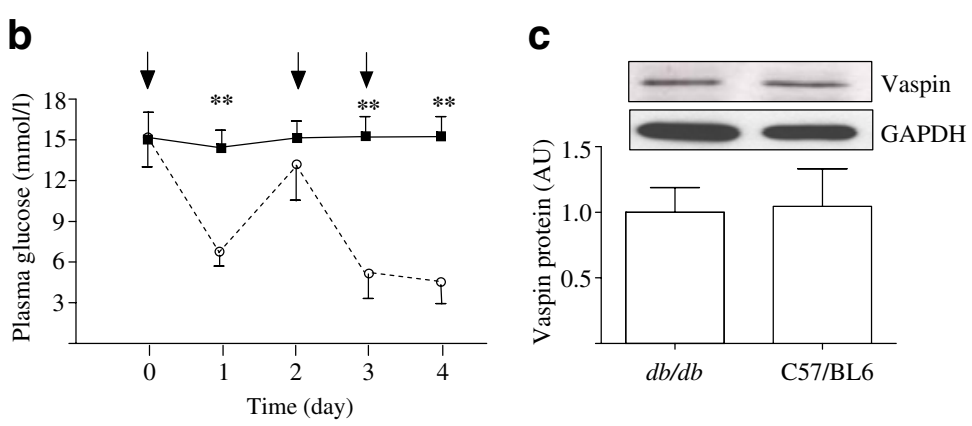

e

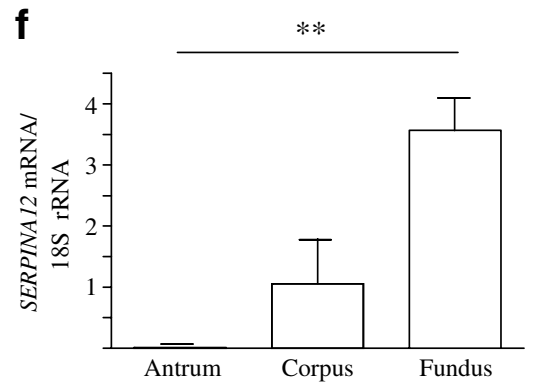

C

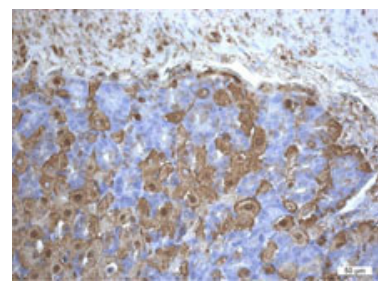

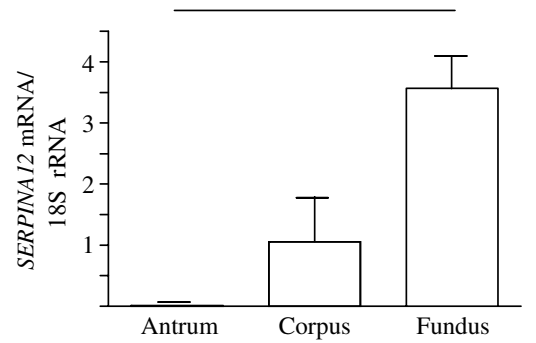

Fig. 1 Effects of peripheral vaspin administration and vaspin production in mouse hypothalamus and human stomach. Fed 11-week-old female $d b / d b$ mice were given i.p. injections of pyrogen-free saline $(n=6)$ or of vaspin $(1 \mathrm{mg} / \mathrm{kg}$ body weight; $n=6)$ at $08: 00 \mathrm{~h}$ on experimental days 1 , 3 and 4. Daily food intake (a) and fed plasma glucose concentrations (b) following intraperitoneal (i.p.) vaspin or saline injection. Black squares, saline; white circles, vaspin. c Vaspin protein production in hypothalamus of $d b / d b(n=5)$ and C57/BL6 $(n=5)$ mice. Representative western blots are shown for vaspin and GAPDH production. d Vaspin

relationships were assessed by least square regression analysis. Statistical software from the SAS Institute (Cary, NC, USA) was used for analyses. A $p$ value of $<0.05$ was considered to be statistically significant.

\section{Results}

Food intake and fed plasma glucose was significantly reduced in i.p. vaspin-treated compared with saline-treated $d b / d b$ mice (Fig. 1a, b). The effects of a single injection are reversed after 1 day without vaspin administration, whereas repeated vaspin injections once a day lead to a sustained reduction in food intake and blood glucose in $d b / d b$ mice (Fig. 1a, b). We investigated whether vaspin may play a role in the central regulation of food intake. First, we found vaspin protein in the hypothalamus of $d b / d b$ and C57BL/6 mice (Fig. 1c) and detected vaspin in the cerebrospinal fluid of five (out of five) healthy individuals who underwent diagnostic lumbar puncture (Fig. 1d). In the stomach explants of five obese patients who underwent gastric sleeve resection, we detected vaspin protein in the gastric mucosa (Fig. 1e), with regional differences in SERPINA12 mRNA expression between the fundus and corpus, but undetectable expression in the antrum (Fig. 1f). concentration in the cerebrospinal fluid of five healthy individuals who underwent diagnostic lumbar puncture. Vaspin was measured in triplicate. e Paraffin section of the mucosa from the stomach of an obese person with normal glucose tolerance who was undergoing gastric sleeve resection. Immunostaining with a monoclonal anti-vaspin antibody (brown, vaspin staining). f Gastric mucosa SERPINA12 mRNA expression in different regions of human stomach after gastric sleeve resection $(n=5) . * *<0.01$

To systematically study the effects of central vaspin administration on food intake, female $d b / d b$ (Fig. 2a-c) and C57BL/6 (Fig. 2d-f) mice were administered an i.c.v. injection of $1 \mu \mathrm{g}$ vaspin and food intake, body weight and fed plasma glucose concentrations were monitored. Central vaspin administration caused a significant temporary reduction in food intake at the first day after i.c.v. injection both in obese $d b / d b$ and lean C57BL/6 mice (Fig. 2a, d). In $d b / d b$, but not in C57BL/6 mice, a single i.c.v. injection of vaspin was sufficient to cause a sustained significant improvement in fed glucose concentrations over at least 6 days (Fig. 2b, e). One i.c.v. vaspin injection did not change body weight, despite the temporary effect on reduced food intake in both lean and obese mice (Fig. 1c, f). Of note, gastric Serpinal 2 mRNA was not affected by i.c.v. or i.p. vaspin treatment in $d b / d b$ or $\mathrm{C} 57 \mathrm{BL} / 6$ mice.

\section{Discussion}

We show here that vaspin is present in the hypothalamus and can be detected in the cerebrospinal fluid of healthy individuals. Both peripheral and central vaspin administration decrease food intake in obese $d b / d b$ and lean C57BL/6 mice. In $d b / d b$, but not $\mathrm{C} 57 \mathrm{BL} / 6$, mice, vaspin treatment 


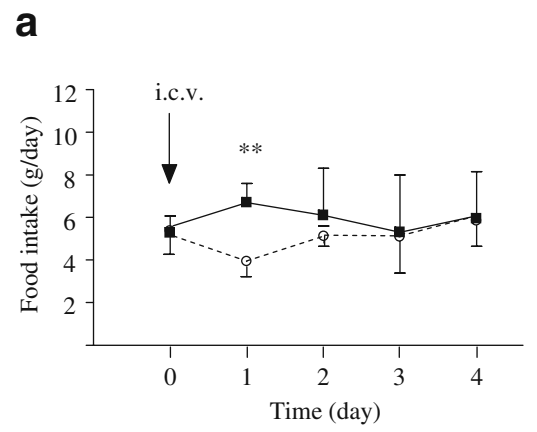

d

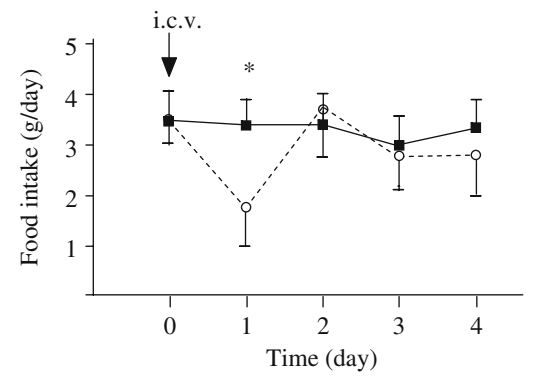

e
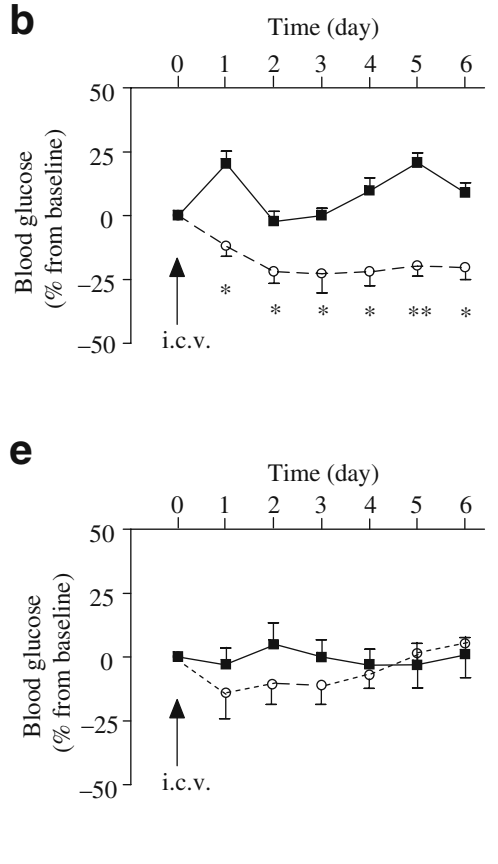

C

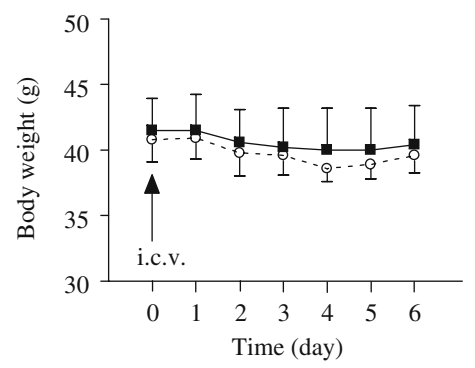

f

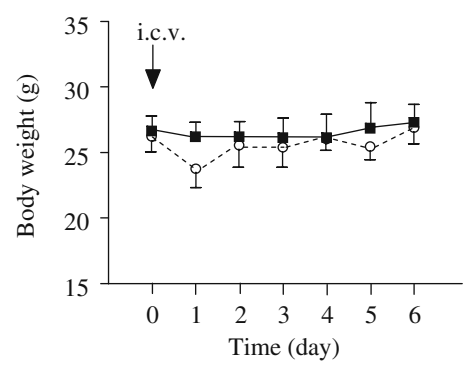

Fig. 2 Potential role of vaspin in the regulation of food intake. Changes in food intake (a, d), fed plasma glucose concentrations (b, e), and body weight (c, f) following a single i.c.v. dose of either artificial cerebrospinal fluid (black squares) or $1 \mu \mathrm{g}$ vaspin (white circles) into

was associated with sustained glucose-lowering effects for at least 6 days after i.c.v. injection. In C57BL/6 mice, i.c.v. vaspin injection did not have significant glucose-lowering effects, despite the reduction in food intake. This result indicates that vaspin lowers plasma glucose (only) in the presence of elevated blood glucose concentrations and may suggest that vaspin treatment does not have the potential to cause hypoglycaemia.

We extend previous findings of gastric vaspin production in mice [5] by demonstrating the presence of vaspin in the gastric mucosa, with regional differences in SERPINA12 mRNA expression, in humans. Increasing the central vaspin concentration may contribute to altered circulating vaspin levels, which may underlie changes in food intake. However, to date, there has been no established method for the measurement of vaspin serum concentrations in mice. Therefore, the hypothesis that i.c.v. vaspin administration causes changes in vaspin serum concentrations needs to be tested in further studies. Vaspin most likely represents a serine protease inhibitor. Although, antiprotease properties have been suggested as a mechanism of action of this protein, to date a protease substrate of vaspin has not been identified. Our finding that both peripheral and central vaspin treatment causes reduced food intake suggests that vaspin inhibits a protease that cleaves a putative anti-orexigenic factor. The effects of vaspin treatment on reduced food intake may therefore be an 11-week-old female $d b / d b(\mathbf{a}-\mathbf{c}, n=8)$ or C57BL6 mice $(\mathbf{d}-\mathbf{f}, n=8)$ up to 6 days after i.c.v. injection. ${ }^{*} p<0.05, * * p<0.01$ for i.c.v. vaspin vs ACSF treatment at the same time point

indirect result of an increase in the stability and efficacy of an anti-orexigenic factor that subsequently mediates vaspin effects to suppress food intake. The observation that food intake returns to normal after 1 day without vaspin treatment suggests that the inhibition of proteinase activity by vaspin is limited by the time taken for elimination of the vaspin-protease complex from the plasma, which is approximately 1 day. Further studies are necessary to unravel the clearance mechanism of the vaspin-protease complex.

In conclusion, our data suggest a previously unrecognised role of vaspin in the regulation of food intake. We are aware that the data from this pilot study are preliminary, but hope that they stimulate further research to elucidate a potential role of vaspin in the control of appetite and satiety. We postulate that vaspin inhibits a protease that degrades an anti-orexigenic factor. Further studies are needed to identify the putative target protease of vaspin.

Acknowledgements This work was supported by grants of the Deutsche Forschungsgemeinschaft, Clinical Research group 'Atherobesity' (KFO152; Be 1264/10-1; BL 833/1-1; KL2346), KO (3880/1-1), DHFD (Diabetes Hilfs- und Forschungsfonds Deutschland; to M. Stumvoll, P. Kovacs, M. Blüher) and Federal Ministry of Education and Research (BMBF), Germany, FKZ: 01EO1001 (N. Klöting).

Duality of interest The authors declare that there is no duality of interest associated with this manuscript. 


\section{References}

1. Hida K, Wada J, Eguchi J et al (2005) Visceral adipose tissue-derived serine protease inhibitor: a unique insulin-sensitizing adipocytokine in obesity. Proc Natl Acad Sci U S A 102:10610-10615

2. Klöting N, Berndt J, Kralisch S et al (2006) Vaspin gene expression in human adipose tissue: association with obesity and type 2 diabetes. Biochem Biophys Res Commun 339:430-436

3. Youn BS, Klöting N, Kratzsch J et al (2008) Serum vaspin concentrations in human obesity and type 2 diabetes. Diabetes $57: 372-377$
4. Kempf K, Rose B, Illig T et al (2010) Vaspin (SERPINA12) genotypes and risk of type 2 diabetes: results from the MONICA/KORA studies. Exp Clin Endocrinol Diabetes 118:184-189

5. Jeong E, Youn BS, Kim DW et al (2010) Circadian rhythm of serum vaspin in healthy male volunteers: relation to meals. J Clin Endocrinol Metab 95:1869-1875

6. Klöting N, Fasshauer M, Dietrich A et al (2010) Insulin-sensitive obesity. Am J Physiol Endocrinol Metab 299:E506-E515

7. Plum L, Ma X, Hampel B et al (2006) Enhanced PIP ${ }_{3}$ signaling in POMC neurons causes $\mathrm{K}_{\mathrm{ATP}}$ channel activation and leads to dietsensitive obesity. J Clin Invest 116:1886-1901 